\title{
Illumina-based transcriptomic profiling of Panax notoginseng in response to arsenic stress
}

Yanfang Liu, Yanhua Mi", Jianhua Zhang, Qiwan Li and Lu Chen

\begin{abstract}
Background: Panax notoginseng, a famous herbal medicine, has recently attracted great attention on its safety and quality since $P$. notoginseng can accumulate and tolerate As from growing environment. For the purpose of understanding As damage to the quality of $P$. notoginseng as well as corresponding tolerance mechanisms, genes involved in As stress response were identified using Illumina sequencing.

Results: Totally 91,979,946 clean reads were generated and were de novo assembled into 172,355 unigenes. A total of 81,575 unigenes were annotated in at least one database for their functions, accounting for $47.34 \%$. By comparative analysis, 1725 differentially expressed genes (DEGs, 763 up-regulated/962 down-regulated) were identified between As stressed plant (HAs) and control plant (CK), among which 20 DEGs were further validated by real-time quantitative PCR (qRT-PCR). In the upstream and downstream steps of biosynthesis pathways of ginsenosides and flavonoids, 7 genes encoding key enzymes were down-regulated in HAs. Such down-regulations were also revealed in pathway enrichment analysis. Genes encoding transporters (transporters of ABC, MATE, sugar, oligopeptide, nitrate), genes related to hormone metabolism (ethylene, ABA, cytokinin) and genes related to arsenic accumulation (HXT, NRAMP, MT and GRX) were differentially expressed. The up-regulated genes included those of oxidative stress-related protein (GSTs, thioredoxin), transcription factors (HSFs, MYBs) and molecular chaperones (HSP).

Conclusions: The down-regulation of biosynthesis of ginsenoside and flavonoid indicated that As accumulation in P. notoginseng can cause not only safety hazard, but also qualitative losses. Aside from the results of arsenic content of seedling roots, the ability of P. notoginseng to over-accumulate arsenic can also be explained by the differential expression of genes of HXT, NRAMP, MT and GRX. To illustrate the detoxification mechanism of P. notoginseng, differential expression of genes encoding oxidative-related proteins, transcription factors, molecular chaperones, transporters and hormone were revealed in our study, which agreed with those reported in Arabidopsis to a certain extent, indicating P. notoginseng and Arabidopsis shared some common detoxification mechanisms in response to As stress. The longer As treatment in our study may account for the smaller quantity of related DEGs and smaller degree of expression differences of certain DEGs compared with those of Arabidopsis.
\end{abstract}

Keywords: Panax notoginseng, Arsenic stress, Illumina sequence, Differential expressed genes (DEGs)

\section{Background}

Panax notoginseng (Burk.) F. H. Chen (Araliaceae) is a perennial herb and has been used as traditional Chinese medicine for thousands of years (Wang et al. 2013; Xia

*Correspondence: zhoumiqu@sina.com; eapvpf2@163.com Quality Standard and Testing Technology Research Institute, Yunnan Academy of Agricultural Sciences, No. 2238, Beijing Road, Kunming 650205, People's Republic of China et al. 2014). Ginsenosides (also called as triterpene saponins) and flavonoid are known as the main pharmacologically active compounds found in P. notoginseng (Ng 2006; Sun et al. 2010). P. notoginseng has been widely consumed as home remedies in both raw (fresh or cooked) and processed (medicinal products) forms (Gong et al. 2015; Wang et al. 2012), and has been attracting more and more attention worldwide due to its anti-oxidative, 
anti-inflammatory, anti-coagulation, neuro-protective, anti-fibrotic, anti-diabetic, anti-cancer as well as antiatherogenic effects (Ng 2006; Sun et al. 2010; Liu et al. 2014). Such increase in popularity has also brought concerns and fears over the quality and safety of the unprocessed products due to the increasing contamination of heavy metals through natural and anthropogenic pathway (Liu et al. 2013a, 2010).

$P$. notoginseng is native to southern China and primarily cultivated in Wenshan, Yunnan province of China, where it occupies $98 \%$ of total yield (Guo et al. 2010; Guo 2007). However, soil in Wenshan has been partly contaminated by As owing to frequent mining activities and large-scale use of As containing pesticide. Different from most plants, toxicity threshold of arsenic to one-year-old seedlings of $P$. notoginseng is $13 \mathrm{mg} \mathrm{kg}^{-1}$ (Mi et al. 2015), while adult plants of $P$. notoginseng can exhibit great tolerance to highly As contaminated environment of up to $250 \mathrm{mg} \mathrm{kg}{ }^{-1}$. Besides, the herb is capable of absorbing As, causing As concentration in root, stem and leaves exceeding the national standard $\left(<2 \mathrm{mg} \mathrm{kg}{ }^{-1}\right)$ significantly (Yan et al. 2012, 2013). Furthermore, flavonoid content of $P$. notoginseng was reported to reduce along with the increase of As accumulation ( $\mathrm{Zu}$ et al. 2014), which also contradicts with most plant species and genus (Su and Zhou 2009). Until now, influence of As stress to the quality of $P$. notoginseng and corresponding tolerance mechanism have been poorly understood.

Transcriptome sequencing provides a cost-effective means of qualitative and quantitative analyses of gene transcripts in many non-model species. In this study, we performed genome-wide transcriptome profiling to identify the genes of $P$. notoginseng responding to As stress to elucidate the quality changes and the tolerance mechanism of $P$. notoginseng in As contaminated conditions.

\section{Methods}

\section{Plant material, treatment of As stress, RNA extraction} and measurement of As content

Seeds of $P$. notoginseng were sown in trays for seedling nursing in January 2014. In October, fifty-two $10-\mathrm{cm}-$ high healthy seedlings were chosen, including four seedlings bred from the same parent plant. After removing soil and rotten/injured roots, the 52 seedlings were transplanted to $500 \mathrm{~mL}$ glass jar filled with $150 \mathrm{~mL}$ nutrient solution (for $P$. notoginseng's exclusive use) (Mi et al. 2015), making sure the roots immersed in solution and the stems aloft. After 14 days, seedlings were divided into two groups: (1) 26 seedlings for control (CK), (2) 26 seedlings for 14 days' exposure experiment: $40 \mathrm{mg} \mathrm{L}^{-1}$ $\mathrm{Na}_{2} \mathrm{HAsO}_{4} \cdot 7 \mathrm{H}_{2} \mathrm{O}$ (HAs). Roots of the 52 seedlings were afterwards rinsed thoroughly with deionized water. The four seedlings sharing the same parent plant were allocated to $\mathrm{CK}$ and HAs groups equally, among which, one root of $\mathrm{CK}$ and one root of HAs were for RNA extraction respectively. Total RNA of each sample was extracted using an E.Z.N.A. Plant RNA Kit (OMEGA Bio-Tec, USA). The quality, purity, concentration and integrity of the RNA samples were assessed accordingly. $3 \mu \mathrm{g}$ RNA per sample was sent to Novogene Bioinformatics Technology CO., LTD, Beijing, China (http://www. novogene.cn) for Illumina sequencing, and the remained was for real-time quantitative PCR (qRT-PCR).

The rest 50 roots were for the purpose of As content comparison. Roots were oven dried at $95{ }^{\circ} \mathrm{C}$ for $30 \mathrm{~min}$, later dried to constant weight at $56^{\circ} \mathrm{C}$ (Mi et al. 2015). Dried roots were pounded into powder and $0.5 \mathrm{~g}$ powder was digested in $\mathrm{HNO}_{3} / \mathrm{HClO}_{4}(10 \mathrm{~mL} / 1 \mathrm{~mL})$ at room temperature for $24 \mathrm{~h}$, then under gradually heating condition from 50 to $150{ }^{\circ} \mathrm{C}$ till white smoke turned up. After cooling, $0.5 \mathrm{~mL} \mathrm{HNO}_{3}$ and deionized water was added to constant volume of $25 \mathrm{~mL}$ (Qiang et al. 2003). Arsenic concentration was determined by inductively coupled plasma mass spectrometry (ICP-MS) (Gao et al. 2015). Data were mean values from five independent biological replicates (five glasses) and each replicate contained five individuals.

\section{Transcriptome library preparation, sequencing, assembly and gene annotation}

Transcription libraries were constructed using Illumina TruSeq $^{\text {TM }}$ RNA Sample Preparation Kit (Illumina, San Diego, USA), and cluster generation were performed using TruSeq PE Cluster Kit v3-cBot-HS (Illumina). The library preparations were then sequenced on an Illumina Hiseq 2000 platform and paired-end reads were generated.

After removing reads containing adapter and ploy-N, as well as low quality reads, clean reads were obtained. Their quality was also ensured via the assessment of Q20, Q30, GC-content and sequence duplication level. Transcriptome assembly was thus accomplished using Trinity (Grabherr et al. 2011).

All the assembled unigenes were searched against the following 7 database, including $\mathrm{Nr}$ (NCBI non-redundant protein sequences), Nt (NCBI non-redundant nucleotide sequences), Pfam (Protein family), KOG/COG (Clusters of Orthologous Groups of proteins), Swiss-Prot (A manually annotated and reviewed protein sequence database), KO (KEGG Ortholog database) and GO (Gene Ontology).

\section{Differential gene expression analysis}

Gene expression levels were measured by RSEM ( $\mathrm{Li}$ and Dewey 2011). For each sequence library, the read counts were adjusted by edge $R$ program package through one 
scaling normalized factor. Differential expression of two samples was analyzed using the DEGseq (Anders and Huber 2010) $R$ package. $Q$ value was used to adjust $P$ value (Storey and Tibshirani 2003). The threshold for significantly differential expression was q-value $<0.005 \& \mid \log _{2}$ (fold change) $\mid>1$.

\section{Enrichment analysis of DEGs}

Gene ontology (GO) enrichment analysis of differential expressed genes (DEGs) was conducted by the GOseq $\mathrm{R}$ package according to Wallenius non-central hyper-geometric distribution (Young et al. 2010), where gene length was taken into account. A corrected $P$ value of $\leq 0.05$ was deemed as a threshold for significant enrichment of the genes. Top GO software was also applied to display enriched GO categories (Alexa and Rahnenfuhrer 2010).

KOBAS software was used to test the statistical enrichment of DEGs in KEGG (Kyoto Encyclopedia of Genes and Genomes) pathway to identify markedly enriched metabolic pathways or signal transduction pathways compared with the whole genome background. A corrected $\mathrm{P}$ value of $\leq 0.05$ was considered as a threshold for significant enrichment of pathways in DEGs.

\section{Quantitative real-time PCR validation}

Twenty genes were selected to carry out Quantitative real-time PCR (qRT-PCR) for the proving of DEGs results of Illumina sequencing. $1 \mu \mathrm{g}$ RNA of CK and HAs were used to reverse-transcribe the first strand cDNA using the PrimeScript ${ }^{\mathrm{TM}}$ RT Reagent Kit (Takara). qRT-PCR was conducted on an optical 96-well plate with an iQ5 multicolor real time PCR system (Bio-RAD, USA). $20 \mu \mathrm{L}$ reaction mixtures contained $1.0 \mu \mathrm{L}$ of cDNA, $10 \mathrm{nM}$ primers and $10 \mu \mathrm{L}$ of $\mathrm{iTaq}^{\mathrm{TM}}$ Universal SYBR Green supermix (BioRAD, USA). The amplification conditions involved an initial step of $30 \mathrm{~s}$ at $95{ }^{\circ} \mathrm{C}$, followed by 40 cycles of $10 \mathrm{~s}$ at $95{ }^{\circ} \mathrm{C}$, and $20 \mathrm{~s}$ at $52{ }^{\circ} \mathrm{C}$ for annealing, and then $30 \mathrm{~s}$ at $72{ }^{\circ} \mathrm{C}$ for extension. For each sample, qPCR was repeated three times with the gene of actin (gene id: comp109453 $\mathrm{c0}$ ) as endogenous control. Software CFX Manaer 2.1 was applied to calculate $\mathrm{Cq}$ value to analyze expressions of actin in CK and HAs. Relative expression level of specific gene was determined as described by Pfaffl (2001).

\section{Results}

\section{Arsenic content and morphological comparison}

After 14 days, seedlings under As stress treatment showed remarkable As accumulation in their roots, with mean content of $26.75 \mathrm{mg} / \mathrm{kg}$, while mean level of arsenic of control (CK) was $0.79 \mathrm{mg} / \mathrm{kg}$ (Table 1). According to paired-samples $t$ test, difference of As content between $\mathrm{CK}$ and HAs was very significant $(P<0.001)$ (Table 1$)$. Arsenic content of $26.75 \mathrm{mg} / \mathrm{kg}$ also indicated P. notoginseng possesses the ability to over-accumulate arsenic.
Table 1 Paired-samples $t$ test of arsenic content of CK and $H A s^{a}$

\begin{tabular}{llllll}
\hline & $\begin{array}{l}\text { Mean } \\
(\mathbf{m g} / \mathbf{k g})\end{array}$ & $\mathbf{N}$ & $\begin{array}{l}\text { Std. } \\
\text { deviation }\end{array}$ & $\begin{array}{l}\text { Std. } \\
\text { error Mean }\end{array}$ & Sig.(2-tailed) \\
\hline $\mathrm{CK}$ & 0.794 & 25 & 0.086 & 0.017 & \\
$\mathrm{HAs}$ & 26.751 & 25 & 1.020 & 0.204 & \\
$\mathrm{CK}-\mathrm{HAs}$ & -25.957 & & 1.036 & 0.207 & $<0.001$
\end{tabular}

a $H$ As seedlings of Panax notoginseng stressed by arsenic

( $40 \mathrm{mg} \mathrm{L}^{-1} \mathrm{Na}_{2} \mathrm{HAsO}_{4} \cdot 7 \mathrm{H}_{2} \mathrm{O}$ ) for 14 days. $\mathrm{CK}$ seedlings for control

Since changes of plant growth were considered as primary symptoms of As-toxicity, morphological traits were also compared between CK and HAs (Fig. 1). Obviously, plants of HAs were seriously dehydrated, their main roots got rotten and fibrous roots were almost absent (Fig. 1).

\section{Sequencing and assembly}

After sequencing, there were totally $105,803,328$ raw reads, 101,043,702 clean reads and 12.64 G clean bases in the two libraries. On average, $95.53 \%$ bases of raw reads had a $Q$ value $\geq 20$ (the percentage of bases with a Phred value $\geq 20$ ) and $91.39 \%$ bases had a $Q$ value $\geq 30$ (the percentage of bases with a Phred value $\geq 30$ ), with an error probability of $0.035 \%$. The GC-contents were $43.42 \%$.

308,350 transcripts were generated using Trinity software, with a mean length of $936 \mathrm{bp}$, N50 of $1719 \mathrm{bp}$ and N90 of 345 bp. 172,355 unigenes were achieved, among which, $71.56 \%$ unigenes $(123,332)$ were $200-500$ bp, $16.51 \%(28,456)$ were $500-1 \mathrm{kbp}, 7.81 \%(13,467)$ were $1-2 \mathrm{kbp}$ and $4.12 \%$ (7100) were $>2 \mathrm{kbp}$.

\section{Annotation and gene expression differences between HAs and CK}

All of the 172,335 assembled unigenes were annotated in databases of Nr, Nt, Swiss-Prot, KO, PFAM, GO, KOG using the BLAST algorithm (E-value $<1 \mathrm{E}-5)$. There were 81,575 unigenes $(47.34 \%)$ annotated in at least one database, and 7867 unigenes (4.56 \%) annotated in all databases. The number of unigenes annotated in databases of $\mathrm{Nr}, \mathrm{Nt}, \mathrm{KO}, \mathrm{PFAM}, \mathrm{GO}$ and KOG were 69,455 (40.29 \%), 22,526 (13.06\%), 26,245 (15.22\%), 52,875 (30.67\%), $55,291(32.07 \%)$ and 32,507 (18.86 \%), respectively. 47,287 (27.43\%) unigenes showed great similarity with unknown genes (hypothetical proteins).

Based on GO classification, 55,291 assembled unigenes were clustered into three functional categories: biological process, cellular component and molecular function. and further classified into 21, 14 and 11 subcategories respectively (Additional file 1: Figure S1). As for KOG functional classification, 32,507 matched unique sequences were classified into 26 categories (Additional file 1: Figure S2). 26,245 assembled unigenes were assigned to the following 


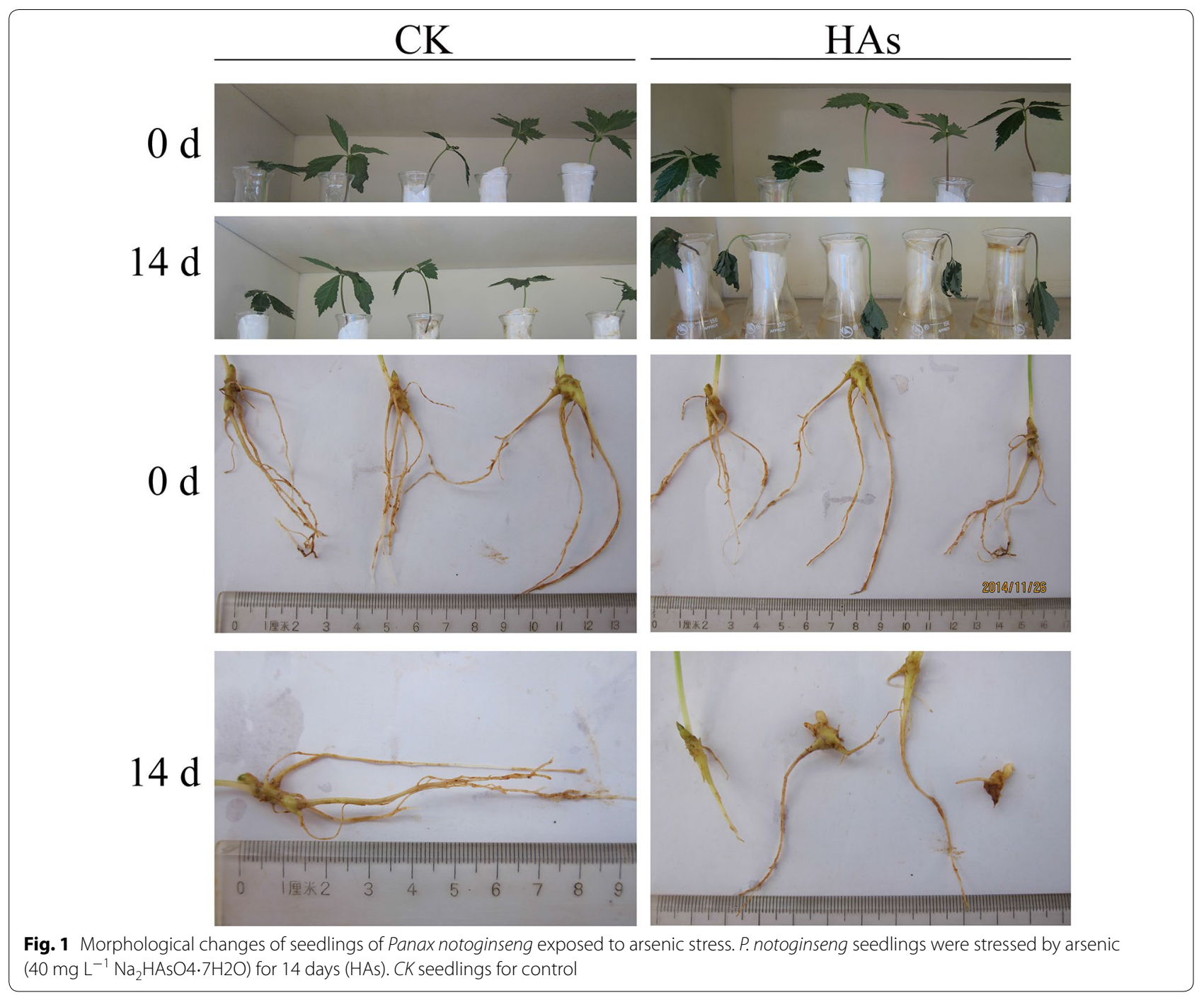

5 KEGG biochemical pathways: metabolism (13,090 unigenes), genetic information processing (8976), organism system (7174), cellular processes (4106) and environmental information processing (3019) (Additional file 1: Figure S3).

According to the criteria [q-value $<0.005$ and $\log _{2}$ (fold change) >1], 1725 genes (accounting for $1.00 \%$ of total unigenes) were determined as significant DEGs between HAs and CK, including 763 up-regulated genes (44.23\% of significant DEGs) and 962 down-regulated genes (55.77 \% of significant DEGs) in HAs (Fig. 2). The $\log _{2}$ fold varied from 1 to 9.18 .

\section{Identification of genes involved in biosynthesis of ginsenosides and flavonoids}

Ginsenosides are synthesized by terpenoid backbone biosynthesis, followed by sesquiterpenoid and triterpenoid biosynthesis (Fig. 3). According to the putative pathway, totally 16 enzymes are involved in ginsenoside biosynthesis where two enzymes (CYP450 s and GTs) participate in the formation of various ginsenosides (Fig. 3). Among our 1725 DEGs, we identified 5 DEGs encoding enzymes involved in above pathway, namely 1 gene (comp129681_ c0) of HMGS (hydroxymethyl glutaryl CoA synthase), 1 gene (comp106407_c0) of HMGR (3-hydroxy-3-methylglutaryl-coenzyme A reductase), 1 gene (comp140511 c0) of DS (Dammarenediol-II synthase) and 2 genes (comp126977_c0 and comp141084_c0) of GTs (glycosyltransferase) (Table 2). Of note, the 5 DEGs were all down regulated and mainly located in the beginning and the end of the pathway, i.e., arsenic stress may inhibit ginsenoside biosynthesis of $P$. notoginseng in the upstream and downstream steps. Such down-regulation was also shown in terpenoid backbone biosynthesis after the 1725 DEGs were likewise searched against KEGG database (Fig. 4). 


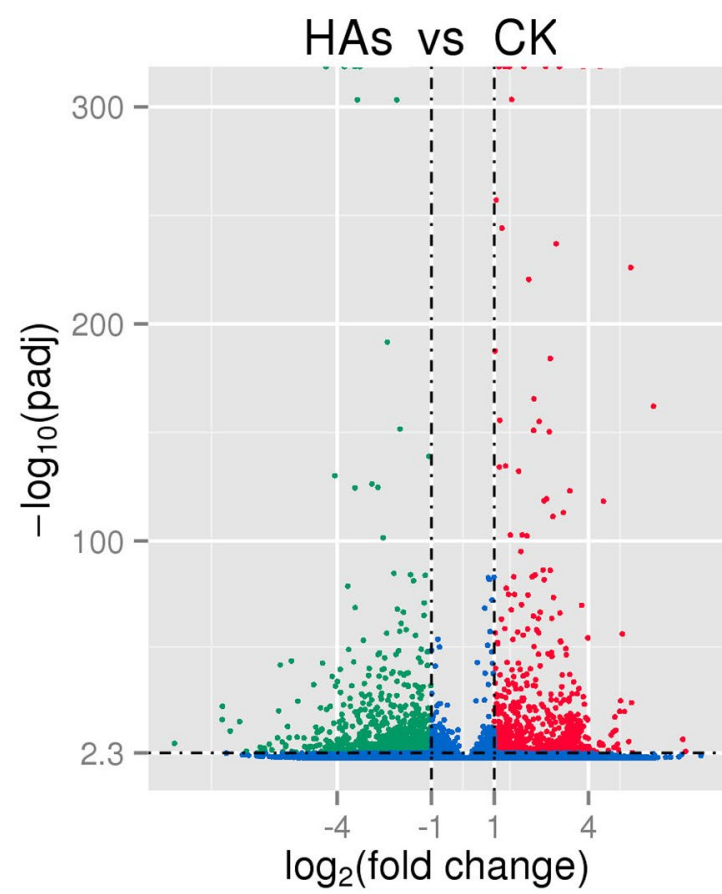

\section{Differential Expressed Genes ( 1725 )}

- up regulated: 763

- down regulated: 962

Fig. 2 Expression patterns of differentially expressed genes (DEGs) identified between HAs and CK. Red and green dots represent DEGs, while blue dots are not DEGs. In total, 1725 unigenes were identified as DEGs (padj < 0.05) between HAs and CK, including 763 up-regulated genes and 962 down-regulated genes in CK. HAs seedlings of Panax notoginseng stressed by arsenic ( $40 \mathrm{mg} \mathrm{L}{ }^{-1} \mathrm{Na}_{2} \mathrm{HAsO}_{4} \cdot 7 \mathrm{H}_{2} \mathrm{O}$ ) for 14 days. CK seedlings for control

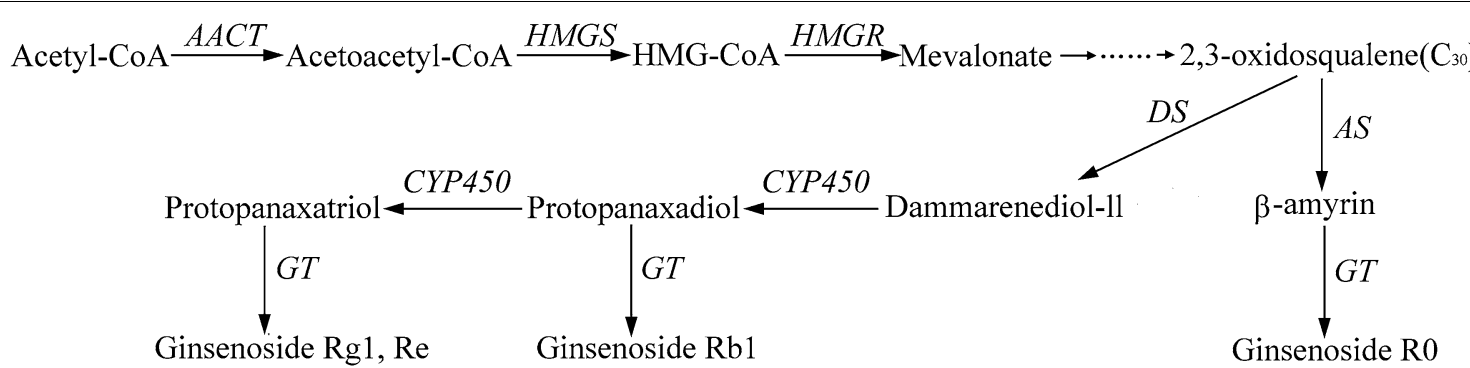

Notoginsenoside R1 Rb2, Rb3, Rc and Rd

Fig. 3 Putative ginsenoside biosynthesis in Panax notoginseng. AACT acetyl-CoA acetyltransferase, HMGS hydroxymethyl glutaryl CoA synthase, HMGR 3-hydroxy-3-methylglutaryl-CoA reductase, AS $\beta$-amyrin synthase, DS dammarenediol-II synthase, CYP450 cytochrome P450, GT glycosyltransferase

Aside from ginsenosides, flavonoid biosynthesis was also depressed. The top three pathways with the most significant degree of DEGs enrichment in KEGG database were 'starch and sucrose metabolism,' 'plant hormone signal transduction' and 'phenylpropanoid biosynthesis' with 29 DEGs, 25 DEGs and 20 DEGs respectively. As a branch of 'phenylpropanoid biosynthesis', 'Flavonoid biosynthesis' enriched 5 differentially expressed genes (Fig. 5). Among the 5 DEGs, 3 genes were up-regulated while the other 2 genes down-regultated. The 3 up-regulated genes were involved in the process of catalyzing Cinnamoyl-CoA to form Caffeoyl-CoA, while the 2 down-regulated genes can directly affect the 'flavone and flavonol biosynthesis' and 'anthocyanin biosynthesis. The 2 down-regulated genes were chalcone synthase (CHS, comp139796_c2, 53.84-fold lower in HAs) and flavonol synthase (FLS, comp78650_c0, 26.87-fold lower in HAs), which also located in the upstream and downstream of the pathway.

qPCR was applied to further validate the expression level of the above 7 down-regulated genes. Results 
Table 2 DEGs between HAs and CK identified by illumina and validated by qRT-PCR

\begin{tabular}{|c|c|c|c|}
\hline Gene ID & Illumina sequencing & QRT-PCR & KO description \\
\hline comp139796_c2 & $-53.83^{\mathrm{a}}$ & -138.82 & chalcone synthase \\
\hline comp78650_c0 & -26.86 & -80.31 & flavonol synthase \\
\hline comp129681_c0 & -3.78 & -9.73 & hydroxymethyl glutaryl CoA synthase \\
\hline comp106407_c0 & -10.00 & -25.62 & 3-hydroxy-3-methylglutaryl-coenzyme A reductase \\
\hline comp140511_c0 & -3.30 & -5.73 & Dammarenediol-II synthase \\
\hline comp126977_c0 & -6.04 & -4.79 & glycosyltransferase \\
\hline comp141084_c0 & -2.13 & -6.34 & glycosyltransferase \\
\hline comp140248_c0 & $6.98^{b}$ & 19.41 & glutathione S-transferase \\
\hline comp117631_c0 & 8.19 & 25.85 & glutathione S-transferase \\
\hline comp128853_c0 & 2.05 & 63.56 & myb proto-oncogene protein, plant \\
\hline comp128641_c0 & 2.32 & 2.17 & myb proto-oncogene protein, plant \\
\hline comp66279_c0 & 3.55 & 2.63 & myb proto-oncogene protein, plant \\
\hline comp128496_c0 & 5.93 & 8.83 & myb proto-oncogene protein, plant \\
\hline comp77739_c0 & 8.52 & 8.26 & myb proto-oncogene protein, plant \\
\hline comp109390_c0 & 6.71 & 5.20 & heat shock transcription factor, other eukaryote \\
\hline comp141438_c1 & 5.22 & 9.27 & heat shock 90 kDa protein \\
\hline comp142366_c1 & 6.33 & 4.68 & heat shock 70 kDa protein \\
\hline comp141020_c0 & 7.02 & 21.39 & heat shock 70 kDa protein \\
\hline comp134360_c1 & 12.85 & 9.71 & heat shock 70 kDa protein \\
\hline comp134360_c0 & 13.58 & 20.37 & heat shock 70 kDa protein \\
\hline
\end{tabular}

HAs seedlings of Panax notoginseng stressed by arsenic $\left(40 \mathrm{mg} \mathrm{L}^{-1} \mathrm{Na}_{2} \mathrm{HAsO}_{4} \cdot 7 \mathrm{H}_{2} \mathrm{O}\right)$ for 14 days. CK seedlings for control. DEGs differentially expressed genes

a Negative values represent the folds of down-regulated expression of genes in $\mathrm{HAs}$ compared with $\mathrm{CK}$

b Positive values represent the folds of up-regulated expression of genes in HAs compared with CK

indicated that, trends of expression differences of these genes detected by qPCR agreed with those concluded by Illumina sequencing (Table 2). Cq values of actin in CK and HAs were 24.66 and 26.93 respectively.

\section{Identification of genes related to tolerance mechanism of $P$. notoginseng to arsenic stress}

As mentioned above, pathways of 'starch and sucrose metabolism' and 'plant hormone signal transduction' enriched the most DEGs in KEGG database. We also subjected genes up- and down-regulated with As exposure to $\mathrm{GO}$ analysis. Up-regulated genes were predominately involved in oxidoreductase activity, gene expression regulation (Additional file 1: Figure S4). Down-regulated genes mainly participated in carbohydrate metabolic process, polysaccharide metabolic process, cellulose metabolic process (Additional file 1: Figure S5). To further revealed the tolerance mechanism of $P$. notoginseng, especially processes of toxicity and detoxification, DEGs involved in arsenic accumulation, transporter, hormone, oxidative stress and transcriptional regulation were investigated.

Totally 7 genes related to arsenic accumulation were differentially expressed, including the up-regulation of 1 gene encoding hexose transporter (HXT) and 1 gene encoding natural resistance-associated macrophage protein (NRAMP), together with the down-regulation of 2 genes encoding Metallothionein (MT) and 3 genes encoding glutaredoxin (GRX). As for transporters, a number of genes encoding transporters were differentially expressed in HAs, including ATP-binding cassette (ABC) transporters, multidrug and toxic compound extrusion (MATE) transporters, as well as other transporters of sugar, oligopeptide and nitrate. As for hormone pathway, ethylene-related genes comprised the largest group of DEGs with the amount of 16 genes, and particularly, 12 ethylene-related genes were up-regulated with As exposure. Besides, DEGs related to hormone metabolism also involved ABA, cytokinin, et al. As for oxidation-reduction, 6 genes encoding thioredoxin and 3 genes encoding glutathione S-transferases (GSTs) were up-regulated in HAs. Besides, a great number of genes encoding transcription factors and molecular chaperones were also up-regulated with As exposure, including heat shock factors (HSF), Myb-related proteins (MYB) and heat shock proteins (HSP).

Based on qPCR verification, trends of expression differences of some above genes agreed with those obtained by Illumina sequencing (Table 2). 
TERPENOID BACKBONE BIOSYNTHESIS

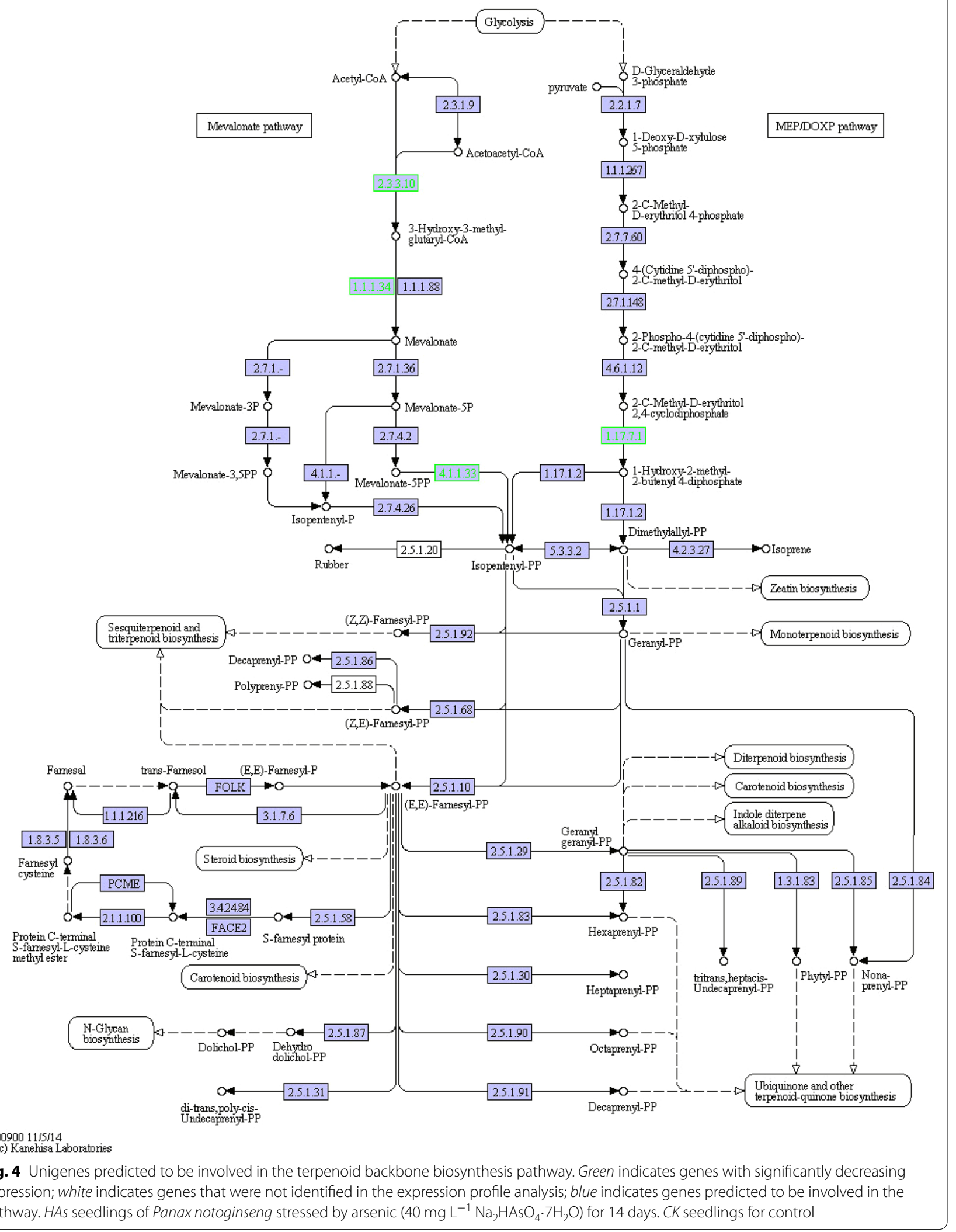


FLAVONOID BIOSYNTHESIS

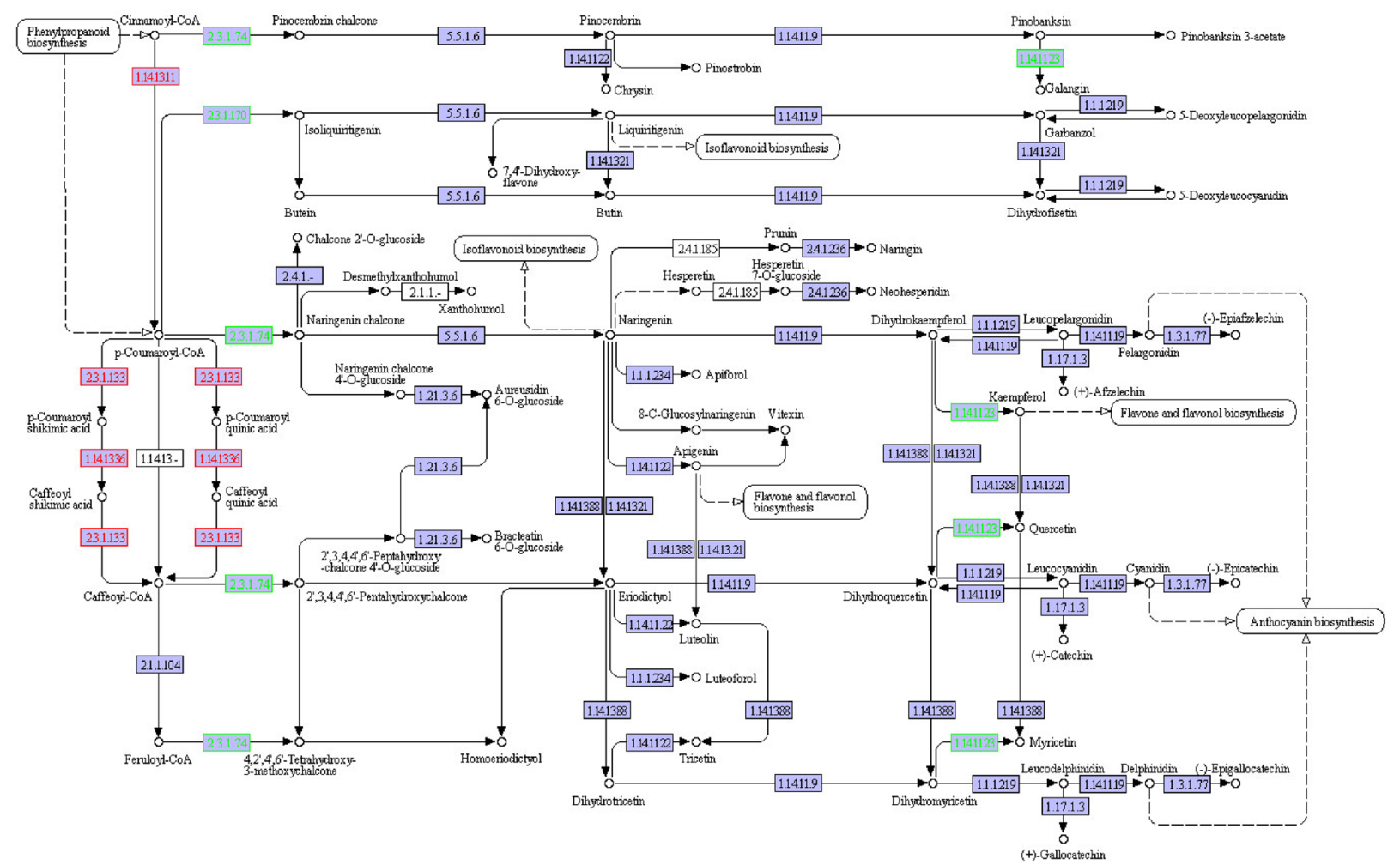

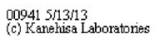

Fig. 5 Unigenes predicted to be involved in the flavonoid biosynthesis pathway. Red indicates genes with significantly increasing expressions in HAs compared with CK; green indicates genes with significantly decreasing expression; white indicates genes that were not identified in the expression profile analysis; blue indicates genes predicted to be involved in the pathway. HAs seedlings of Panax notoginseng stressed by arsenic (40 mg. $\mathrm{L}^{-1} \mathrm{Na}_{2} \mathrm{HAsO}_{4} \cdot 7 \mathrm{H}_{2} \mathrm{O}$ ) for 14 days. CK seedlings for control

\section{Discussion}

Root growth inhibition is the primary response of the plant exposed to arsenic and it is the As-sensitivity of the root that limits the productivity of the entire plant (Fu et al. 2014). Our study showed that, 14-day treatment of arsenic $\left(40 \mathrm{mg} \mathrm{L}{ }^{-1} \mathrm{Na}_{2} \mathrm{HAsO}_{4} \cdot 7 \mathrm{H}_{2} \mathrm{O}\right.$ ) could seriously damage the root growth of $P$. notoginseng, thus making the plants dehydrated.

Identification of genes involved in ginsenosides of $P$. notoginseng (Liu et al. 2015) provided us an insight to reveal the impact of arsenic accumulation to the quality of $P$. notoginseng. Our root transcriptomes showed that, As stress could reduce biosynthesis of ginsenoside and flavonoid by inhibiting gene expressions in the upstream and downstream steps of the pathways, indicating arsenic accumulation in $P$. notoginseng can cause qualitative losses, aside from the safety hazard reported previously (Liu et al. 2013a, 2010).

Flavonoid plays important roles in protecting organisms against biotic and abiotic stresses via eliminating reactive oxygen species (ROS) (Kumar et al. 2010; Liu et al. 2013b). Arsenic stress can induce the biosynthesis of flavonoid in Sarcandra glabra (Thunb) (Su and Zhou 2009), indicating flavonoid would be involved in positive response to As stress. However, our researches showed that, genes encoding chalcone synthase (CHS, comp139796_c2) and flavonol synthase (FLS, comp78650_c0), two key enzymes of flavonoid synthesis, were down-regulated in P. notoginseng under As stress. Such result contradicts previous findings obtained from other plant species and genus, but it corresponds well with an earlier report revealing the significant negative relationship between flavonoid content and As accumulation in P. notoginseng ( $\mathrm{Zu}$ et al. 2014). Therefore, $P$. notoginseng may have different mechanism of As response, e.g., without resorting to flavonoid, and there may be other alternatives contributing to As response.

Tolerance mechanisms of plant to arsenic involve As accumuation and detoxification (Kumar et al. 2015). Therefore, genes related to As accumulation, transporter 
and hormone pathways may be As-tolerance associated (Fu et al. 2014; Kumar et al. 2015). In our study, genes encoding HXT and NRAMP were up-regulated and up-regulation of these genes can increase As accumulation (Shah et al. 2010; Tiwari et al. 2014), while genes encoding MT and GRX were down-regulated and upregulation of these genes can lead to the decrease of As accumulation (Grispen et al. 2009; Sundaram et al. 2009). These results are good explanations to the ability of $P$. notoginseng to over-accumulate arsenic. Besides, genes encoding transporters (e.g., transporters of ABC, MATE, sugar, oligopeptide, nitrate, et al.) and genes related to hormone metabolism (ethylene, ABA, cytokinin) were differentially expressed, which also corresponded with those in Arabidopsis (Fu et al. 2014). Although P. notoginseng and Arabidopsis shared some common mechanisms in response to As stress, the quantity of As-tolerance associated genes (especially those involved in pathways mentioned above) was not as large as those revealed in Arabidopsis, and the degrees of expression differences of those DEGs were also smaller as a whole than those in Arabidopsis (Fu et al. 2014). Aside from genotype variation, treatment variation may also account for the differences. Compared with the research of Arabidopsis, duration of As exposure in our study was 14 days, much longer than 2 days. Therefore, it was the late stage of $P$. notoginseng to survive in As stressful condition that our transcriptional profiling revealed. The late stage can also be verified by the rotten roots and dehydrated plant of HAs. In this stage, As-tolerance associated genes may not as sensitive and active as those in early stage.

Glutathione S-transferases (GSTs) and thioredoxin are principally known for their role in detoxification reactions (Board and Menon 2013). Transgenic Arabidopsis plants over-expressing OsGSTL2 (GST from rice) show an increase in tolerance to arsenic exposure (Kumar et al. 2013). In our study, up-regulation of GSTs and thioredoxin in HAs indicated GSTs and thioredoxin can develop plants with improved detoxification mechanism under As stress. Some isoforms of GST show dual activity, additionally functioning as a glutathione peroxidase in the presence of reactive oxygen species (Marrs 1996). Among the 3 up-regulated GSTs in our study, one belongs to Tau subfamily. The up-regulation of Tau class GSTs has also been noted in transcriptomic and proteomic analysis of plant roots under As stress (Norton et al. 2008; Ahsan et al. 2008). In addition, a great number of genes encoding transcription factors (TFs) and molecular chaperones, e.g. HSF, MYB and HSP, were also up-regulated under As treatment, indicating gene regulation at transcriptional level is the key mechanism of $P$. notoginseng to tolerate toxicity of As. The up-regulations of oxidative stress-related genes, TF genes and molecular chaperone genes in our study corresponded with those in Arabidopsis (Fu et al. 2014), indicating P. notoginseng and Arabidopsis take advantage of antioxidant and transcriptional regulation system to cope with the stressful condition.

MYB family and HSF family participate in various biological processes, including regulation of primary and secondary metabolism, defense and stress responses ( $\mathrm{Li}$ et al. 2014, 2013c). There were multiple unigenes annotated to the same protein, which may represent different members of the same gene family. Plant HSFs are assigned to 3 classes (A, B and C) (Liu et al. 2013c). The up-regulated HsfB3 (comp109390_c0) in our study belongs to class B, which lacks AHA (aromatic, large hydrophobic, and acidic amino acid residues) in the activation domain. HSPs are known as molecular chaperones, essential for the survival of cells exposed to various stresses owing to their functions in folding, trafficking, maturation and degradation of proteins (Liu et al. 2013c). The 5 up-regulated HSPs in our study included 4 HSP70 and 1 HSP90. Consistent with our finding, arsenic can lead to up-regulation of HSP70 and HSP90 in Labeo rohita fingerlings (Banerjee et al. 2015), and expression of HSP70 can be induced by arsenic trioxide in MDA231 cells (Kim et al. 2005). Therefore, HSP70 and HSP90 would contribute to As response. Since HSPs are expressed under the regulation of HSFs at transcriptional level (Liu et al. 2013c), the above 5 up-regulated HSP genes might be supposed as target genes of HsfB3 under As stress in P. notoginseng. However, the underlying mechanism of the regulation is now an open question demanding further researches.

\section{Conclusions}

Aside from safety hazard reported previously, our study found that arsenic accumulation can cause qualitative losses of roots of $P$. notoginseng, where biosynthesis of ginsenoside and flavonoid were depressed. Without resorting to flavonoid to fight against arsenic, $P$. notoginseng turned to oxidative stress-related proteins (GSTs, thioredoxin), transcription factors (HSFs, MYBs), molecular chaperones (HSPs), as well as transporter system (transporters of ABC, MATE, sugar, oligopeptide, nitrate) and hormone pathway (ethylene, ABA cytokinin) for survival. P. notoginseng and Arabidopsis shared some common mechanisms in response to As stress, but the quantity of related DEGs and the degrees of expression differences of specific DEGs were not as large as those in Arabidopsis (Fu et al. 2014). Aside from genotype variation, variation in duration of As exposure may also account for the differences. 


\section{Additional file}

Additional file 1: Figure S1. Gene Ontology classification of matched unigenes. 55,291 GO annotated unigenes were classified into 3 functional categories: biological process, cellular component and molecular function. Figure S2. KOG functional classification of matched unigenes. 32,507 KOG annotated unigenes were clustered into 26 categories. Figure S3. KEGG classification of assembled unigenes. 26,245 unigenes were assigned to 5 KEGG biochemical pathways: metabolism, genetic information processing, organism system, cellular processes and environmental information processing. Figure S4. Enriched GO terms of upregulated genes. Genes up-regulated with As exposure were subjected to $\mathrm{GO}$ analysis. Figure S5. Enriched $\mathrm{GO}$ terms of down-regulated genes. Genes down-regulated with As exposure were subjected to $\mathrm{GO}$ analysis.

\section{Authors' contributions}

YHM originally formulated the idea and developed methodology. YFL and QWL wrote the manuscript with inputs from other authors. YFL, JHZ and LC performed data analyses. All authors read and approved the final manuscript.

\section{Acknowledgements}

This work was supported by Grants from the National Natural Science Foundation of China (NSFC) (21267024), Natural Science Foundation for Youth of Yunnan Province (2014FD063), Personnel Training Project of Yunnan Province (2014HB059) and Team Training Project of Yunnan Province (2015HC025).

\section{Competing interests}

The authors declare that they have no competing interests.

Received: 4 October 2015 Accepted: 5 May 2016

Published online: 02 June 2016

\section{References}

Ahsan N, Lee DG, Alam I, Kim PJ, Lee JJ, Ahn YO, Kwak SS, Lee IJ, Bahk JD, Kang KY, Renaut J, Komatsu S, Lee BH (2008) Comparative proteomic study of arsenic induced differentially expressed proteins in rice roots reveals glutathione plays a central role during As stress. Proteomics 8:3561-3576

Alexa A, Rahnenfuhrer J (2010) TopGO: enrichment analysis for gene ontology. R Packag Version 2:12

Anders S, Huber W (2010) Differential expression analysis for sequence count data. Genome Biol. doi:10.1186/gb-2010-11-10-r106

Banerjee S, Mitra T, Purohit GK, Mohanty S, Mohanty BP (2015) Immunomodulatory effect of arsenic on cytokine and HSP gene expression in Labeo rohita fingerlings. Fish Shellfish Immunol 44(1):43-49

Board PG, Menon D (1830) Glutathione transferases, regulators of cellular metabolism and physiology. Biochim Biophys Acta 5:3267-3288

Fu SF, Chen PY, Nguyen QTT, Huang LY, Zeng GR, Huang TL, Lin CY, Huang HJ (2014) Transcriptome profiling of genes and pathways associated with arsenic toxicity and toleranc in Arabidopsis. BMC Plant Biol. doi:10.1186/1471-2229-14-94

Gao ZJ, Gao HX, Liu YL, Liu N (2015) Contents determination of 5 heavy metals in 5 TCM powders by ICP-MS. Chin Pharm 26(27):3847-3849 (in Chinese)

Gong XC, Chen HL, Pan JY, Qu HB (2015) Optimization of Panax notoginseng extraction process using a design space approach. Sep Purif Technol 141:197-206

Grabherr MG, Haas BJ, Yassour M, Levin JZ, Thompson DA, Amit I, Adiconis X, Fan L, Raychowdhury R, Zeng QD, Chen ZH, Mauceli E, Hacohen N, Gnirke A, Rhind N, Palma FD, Birren BW, Nusbaum C, Lindblad-Toh K, Friedman N, Regev A (2011) Full-length transcriptome assembly from RNA-Seq data without a reference genome. Nat Biotechnol 29:644-652

Grispen VMJ, Irtelli B, Hakvoorta HJ, Vooijs R, Bliek B, Bookum WM, Verkleij JAC, Schat H (2009) Expression of the Arabidopsis metallothionein 2b enhances arsenite sensitivity and root to shoot translocation in tobacco. Environ Exp Bot 66:69-73
Guo XC (2007) New green industry — takes Wenshan Panax notoginseng status and its future as an example. Ecol Econ 1:114-117

Guo HB, Cui XM, An N, Cai GP (2010) Sanchi ginseng (Panax notoginseng (Burkill) F. H. Chen) in China: distribution, cultivation and variations. Genet Resour Crop Evol 57:453-460

Kim YH, Park EJ, Han ST, Park JW, Kwon TK (2005) Arsenic trioxide induces Hsp70 expression via reactive oxygen species and JNK pathway in MDA231 cells. Life Sci 77(22):2783-2793

Kumar R, Tayade A, Chaurasia O, Sunil H, Singh SB (2010) Evaluation of antioxidant activities and total phenol and flavonoid content of the hydroalcoholic extracts of Rhodiola sp. Phcog J 2(11):431-435

Kumar S, Asif MH, Chakrabarty D, Tripathi RD, Dubey RS, Trivedi PK (2013) Expression of a rice Lambda class of glutathione S-transferase, OSGSTL2, in Arabidopsis provides tolerance to heavy metal and other abiotic stresses. J Hazard Mater 248-249:228-237

Kumar S, Dubey RS, Tripathi RD, Chakrabarty D, Trivedi PK (2015) Omics and biotechnology of arsenic stress and detoxification in plants: current updates and prospective. Environ Int 74:221-230

Li B, Dewey CN (2011) RSEM: accurate transcript quantification from RNA-Seq data with or without a reference genome. BMC Bioinform. doi:10.1186/1471-2105-12-323

Li CN, Ng CKY, Fan LM (2014) MYB transcription factors, active players in abiotic stress signaling. Environ Exp Bot 2823:1-12

Liu XJ, Liu WJ, Lin AJ, Liu YX (2010) Survey of arsenic concentrations in Chinese herbal medicines (CHMs) and preliminary risk assessment of As in CHMs on human health. Huan Jing Ke Xue 31(12):3036-3042 (in Chinese)

Liu XJ, Zhao QL, Sun GX, Williams P, Lu XJ, Cai JZ, Liu WJ (2013a) Arsenic speciation in Chinese herbal medicines and human health implication for inorganic arsenic. Environ Pollut 172:149-154

Liu ML, Li XR, Liu YB, Cao B (2013b) Regulation of flavanone 3-hydroxylase gene involved in the flavonoid biosynthesis pathway in response to UV-B radiation and drought stress in the desert plant, Reaumuria soongorica. Plant Physiol Biochem 73:161-167

Liu YF, Zhang CX, Chen J, Guo LH, Li XL, Li WP, Yu ZF, Deng JS, Zhang PY, Zhang KQ, Zhang LM (2013c) Arabidopsis heat shock factor HsfA1a directly senses heat stress, $\mathrm{pH}$ changes, and hydrogen peroxide via the engagement of redox state. Plant Physiol Biochem 64:92-98

Liu J, Wang Y, Qiu L, Yu Y, Wang C (2014) Saponins of Panax notoginseng: chemistry, cellular targets and therapeutic opportunities in cardiovascular diseases. Expert Opin Investig Drugs 23:523-539

Liu MH, Yang BR, Cheung WF, Yang KY, Zhou HF, Kwok JSL, Lui GC, Li XF, Zhong SL, Lee SMY, Tsui SKW (2015) Transcriptome analysis of leaves, roots and flowers of Panax notoginseng identifies genes involved in ginsenoside and alkaloid biosynthesis. BMC Genom. doi:10.1186/s12864-015-1477-5

Marrs KA (1996) The functions and regulation of glutathione S-transferases in plants. Annu Rev Plant Physiol Plant Mol Biol 47:127-158

Mi YH, Li QW, Liu DH, Wang LX, Chen L, Ma J, Zhang X (2015) Toxicity and its threshold of arsenic to Panax notoginseng seedlings. Environ Sci Technol 38(7):10-16 (in Chinese)

Ng TB (2006) Pharmacological activity of sanchi ginseng (P. notoginseng). J Pharm Pharmacol 58(8):1007-1019

Norton GJ, Lou-Hing DE, Meharg AA, Price AH (2008) Rice-arsenate interactions in hydroponics: whole genome transcriptional analysis. J Exp Bot 59:2267-2276

Pfaffl MW (2001) A new mathematical model for relative quantification realtime RT-PCR. Nucleic Acids Res 29(9):2002-2007

Qiang WG, Yang HF, Mao H, Yan J (2003) Determination of total arsenic and abio-arsenic in foods. National Handbook GB/T 5009.11-2003. People's Republic of China, Beijing (in Chinese)

Shah D, Shen MWY, Chen W, Da Silva NA (2010) Enhanced arsenic accumulation in Saccharomyces cerevisiae overexpressing transporters Fps $1 p$ or Hxt7p. J Biotechnol 150:101-107

Storey JD, Tibshirani R (2003) Statistical significance for genomewide studies. Proc Nati Acad Sci USA 100(16):9440-9445

Su H, Zhou CL (2009) Influence of different adversity stress on total flavones content in Sarcandra glabra (Thunb) Nakai. J Anhui Agric Sci 37(17):7995 (in Chinese)

Sun S, Wang CZ, Tong R, Li XL, Fishbein A, Wang Q, He TC, Du W, Yuan CS (2010) Effects of steaming the root of Panax notoginseng on chemical composition and anticancer activities. Food Chem 118:307-314 
Sundaram S, Wu S, Ma LQ, Rathinasabapathi B (2009) Expression of a Pteris vittata glutaredoxin PVGRX5 in transgenic Arabidopsis thaliana increases plant arsenic tolerance and decreases arsenic accumulation in the leaves. Plant Cell Environ 32:851-858

Tiwari M, Sharma D, Dwivedi S, Singh M, Tripathi RD, Trivedi PK (2014) Expression in Arabidopsis and cellular localization reveal involvement of rice NRAMP, OsNRAMP1, in arsenic transport and tolerance. Plant Cell Environ 37:140-152

Wang D, Liao PY, Zhu HT, Chen KK, Xu M, Zhang YJ, Yang CR (2012) The processing of Panax notoginseng and transformation of its saponin components. Food Chem 132:1808-1813

Wang D, Koh HL, Hong Y, Zhu HT, Xu M, Zhang YJ, Yang CR (2013) Chemical and morphological variations of Panax notoginseng and their relationship. Phytochemistry 93:88-95

Xia P, Guo H, Liang Z, Cui X, Liu Y, Liu F (2014) Nutritional composition of Sanchi (Panax notoginseng) seed and its potential for industrial use. Genet Resour Crop Evol 61:663-667
Yan XL, Lin LY, Liao XY, Zhang WB (2012) Arsenic accumulation and resistance mechanism in Panax notoginseng, a traditional rare medicinal herb. Chemosphere 87:31-36

Yan XL, Lin LY, Liao XY, Zhang WB, Wen Y (2013) Arsenic stabilization by zerovalent iron, bauxite residue, and zeolite at a contaminated site planting Panax notoginseng. Chemosphere 93:661-667

Young MD, Wakefield MJ, Smyth GK, Oshlack A (2010) Gene ontology analysis for RNA-seq: accounting for selection bias. Genome Biol. doi:10.1186/ gb-2010-11-2-r14

Zu YQ, Sun JJ, Min Q, Li ZR, Feng GQ, Li Y (2014) Response of flavonoid content in Panax notoginseng to As and its enzymological mechanism. Chin J Appl Environ Biol 20(6):1005-1010 (in Chinese)

\section{Submit your manuscript to a SpringerOpen ${ }^{\circ}$ journal and benefit from:}

- Convenient online submission

- Rigorous peer review

- Immediate publication on acceptance

- Open access: articles freely available online

- High visibility within the field

- Retaining the copyright to your article 